\title{
Approach to the evaluation of the functional state of the human body taking into account the variability of medical and biological indicators
}

\author{
O. B. Ivanets, \\ Department of Biocybernetics and \\ Aerospace Medicine, \\ National Aviation University \\ Kyiv, Ukraine; \\ olchik2104@ukr.net
}

\author{
L.O. Kosheva, \\ Department of Biocybernetics and \\ Aerospace Medicine, \\ National Aviation University \\ Kyiv, Ukraine; \\ 1.kosh@ukr.net
}

\begin{abstract}
The approach to the evaluation of the functional state of the human body as a system with a set of interrelated functional subsystems of the homeostatic type is proposed. The approach is considered on the basis of an assessment of the state of the respiratory subsystem, which can be described by a complex of statistically stable medical and biological indicators. Indicators obtained during monitoring of the state of polar explorers of the XXI Antarctic expedition at the station "Academician Vernadsky" in difficult conditions that can be attributed to the extreme. It was established that when forming the rules for making decisions on the suitability of winterers for further staying in the conditions of the action of extreme factors it is necessary to consider not only the values of the indicators of the state of the subsystems of the organism, but also their accuracy parameters. The analysis of the uncertainty of the obtained indicators by the proposed information model is carried out. It is shown that in this case it is necessary to take into account the features of a biological object, which has an individual and group variation.
\end{abstract}

Keywords - biological object, functional state, homeostasis, integral index, system of organism, decision making, variability, uncertainty

\section{INTRODUCTION}

One of the most important tasks in the field of health is the development of technologies for assessing the functional state of the body. The analysis of the functional state of the body is necessary for the correct conclusion when diagnosing diseases, analyzing the current functional state, choosing therapeutic measures, resolving rehabilitation issues, providing conditions for maintaining life while performing professional duties in extreme conditions, evaluating professional fitness for a certain type of activity and many other tasks.

In recent years, interest has increased in the problems of building systems for medical diagnostics, the methodology for analyzing medical information, and mathematical models for diagnosing and predicting various functional states $[1,2]$.

Investigating the peculiarities of functioning and determining the role of each of the subsystems and their interactions will increase the reliability of the evaluation of the functional state of the whole organism. The analysis of the ways of the variability of the parameters of the whole organism as a changing biological object, taking into account the technical features (instrumental uncertainty) of the diagnostic apparatus and diagnostic methods, is a complex task. Therefore, in the framework of the work, for example, one of the subsystems of the body is considered the respiratory subsystem.

\section{PROBLEM RESOLUTION}

As an object of research, the functional state of the members of the Antarctic expedition, which for 10 months is at the station "Academician Vernadsky" for carrying out scientific researches $[3,4]$, is chosen.

The task is to prognosis the state of the organism in order to determine the suitability of winterers for further stay under the conditions of extreme factors. For this object of research, constant observation of changes in physiological parameters is carried out. In order to increase the reliability of decisionmaking in the elaboration of the results of observations, it is necessary to take into account both the own variability of the indicators of the state of the organism and the uncertainty of observations of the diagnostic equipment, caused by both the technical state of the equipment, and the imperfection of the method of conducting the research.

\section{FEATURE OF THE BIOLOGICAL}

In order to analyze a large amount of information coming from a biological research object, it is necessary to understand its features. Different objects act as biological: living organism and its environment, organ and group of organs, physiological system and biological feedback, cell and cell group, blood and blood filling, and so on. Biological object as a special object of research is described by a complex of medical and biological indicators - groups of physical, biochemical, psychological parameters that are determined in the course of research $[5,6]$.

Getting a complete description of the biological object, and the human body in particular, is practically impossible. Modern biology and medicine are not able to give all the information about the structure and functioning of all kinds of biological objects. Due to the lack of studying of the biological object it is difficult to obtain adequate mathematical models between the registered parameters and the corresponding biological indicators that characterize the time-spatial state of the biological object [7, 8].

Almost all characteristics of a biological object are characterized by variability - a specific and distinctive feature of all biomedical measurements. Variation of attribute occurs if its value varies from individual to individual or for one individual in time. Almost always it is impossible to carry out repeated control measurements of the parameters of the same biological object, as is customary in the technique, which leads to great difficulties in the estimation of random 
variables in the measurement and, as a consequence, errors in the diagnosis. The variability and individual spread of the parameters of a biological object, their interconnection, nonlinearity of these bonds, the presence of a high level of interference, makes the task of an objective assessment of the state of a biological object rather complicated. In the biological experiment, there is no notion of "true value", only the mean, and the spread of data, due to the individual reaction of the organism, can vary greatly.

In the process of measurement, the medical and biological parameters of a biological object may change with long measurements and due to psycho-physiological factors (especially in Antarctica).

Thus, a living organism is a set of interrelated functional systems of homeostatic type that can be described by a complex of statistically stable medical and biological indicators.

\section{STATE OF HOMEOSTASIS}

The above features of a biological object as an object of research can be overcome if, as a methodological basis of research, take homeostasis of a biological object, that is its ability to provide stability of structure, elemental composition, functions performed, maintenance of characteristic parameters in vital limits independently from changes in environmental conditions [9]. At the expense of homeostasis, the body maintains an organism's body in a state of equilibrium for the ability to perform professional duties.

In the work of the body of the participant of the expedition is considered as a holistic system, which is affected by destabilizing factors of various kinds. The figure 1 shows the influential factors on the state of the organism.

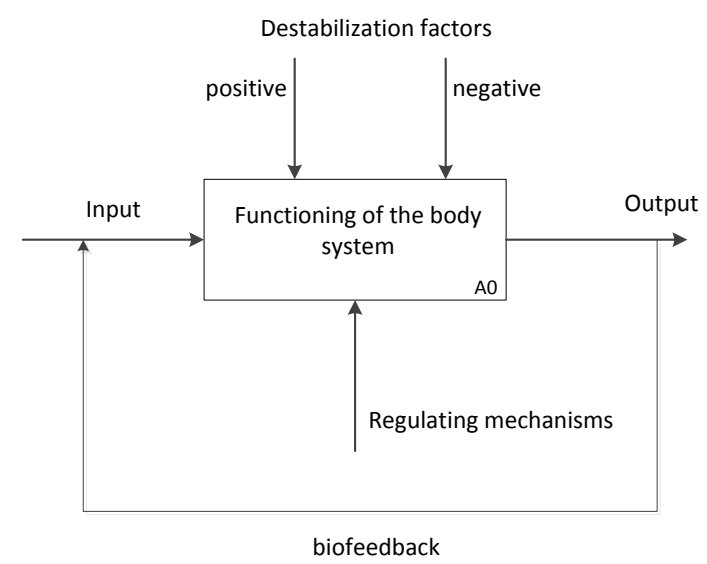

Figure 1. Influence of factors on the state of the organism

For this system, it is suggested that a decomposition be performed, as shown in Figure 2.

The state of human homeostasis is supported by the functioning of the following subsystems: cardiovascular, respiratory, urinary tract, musculoskeletal. Nervous and endocrine systems perform functions of management (regulation) to provide a state of homeostasis. Consideration of the influence of the variability of the state of all subsystems of the organism on the human homeostasis within the article is impossible, therefore only the component of uncertainty associated with a separate respiratory subsystem is considered in the work.

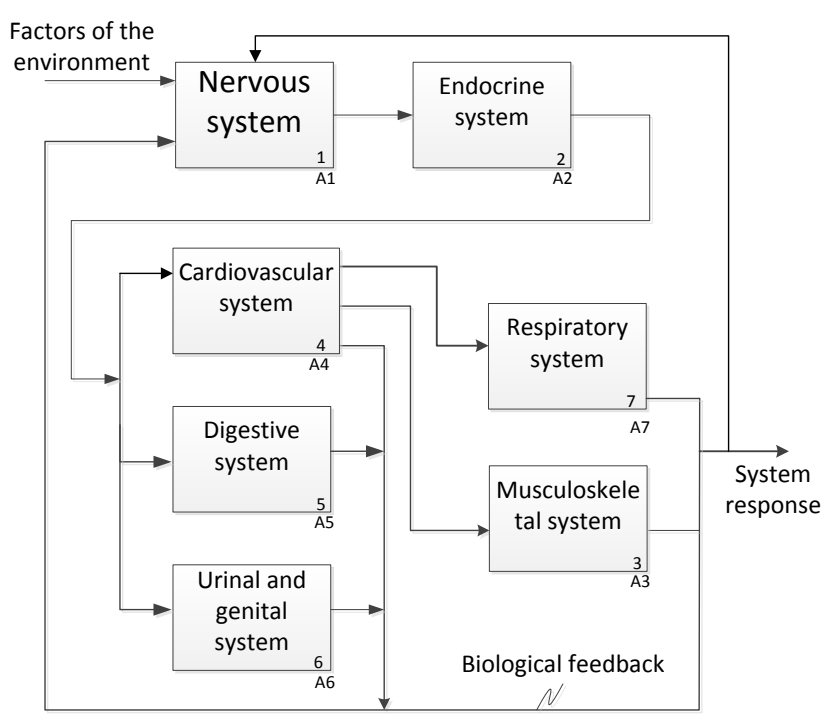

Figure 2. Decomposition of the body system

In general, as a result of measuring the indicators of the state of a biological object, they receive a set of their individual assessments. It is impossible to compare and analyze simultaneously all the individual assessments, and it is inappropriate, because there is always a spread of them, due to the uncontrolled difference of the objects of the experiment (each subject is unique). Therefore, in order to obtain the quantity available for review and smooth individual vibrations, use so-called aggregated (collective, group, derivative) estimates $[10,11]$. Obtaining aggregated estimates based on individual ones is their transformation, which is a separate task. Due to the imperfection of the methods of mathematical analysis, which is not adapted to study such volatile objects as biological, the generalization of the results of the study leads to difficulties in the derivation of the aggregated (integral) indicator.

The following equation is proposed as an integral indicator of the assessment of the state of the respiratory subsystem [12]:

$$
Y=100 * \frac{A * B-C * D}{A}
$$

where $\mathrm{A}$ - maximum ventilation of the lungs;

$\mathrm{B}$ - index of hypoxia,

C - Skibinskaya index,

D - Harrison Ventilation Index.

Figure 3 proposes a model for evaluating the functional state of the respiratory subsystem based on the integral index.

According to model (1), the ventilation index is calculated by the formula:

$$
D=\frac{x_{2}}{x_{0}}
$$

where $x_{2}$ - minute volume of breath;

$x_{0}$ - actual living capacity of the lungs.

The value of the ventilation index can be defined as a criterion for realizing the potential capabilities of the external breathing system of a particular individual. 


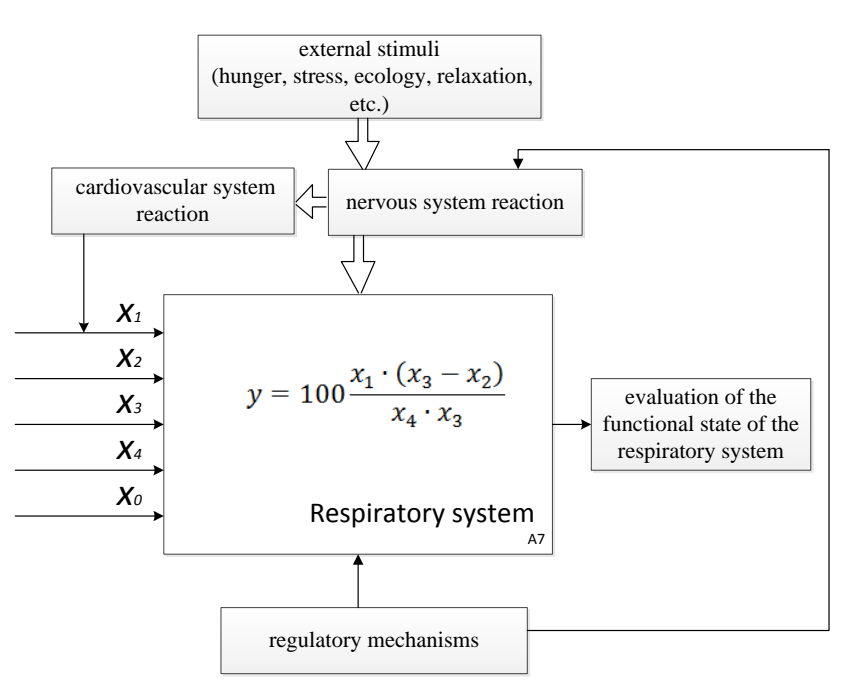

Figure 3. Model for evaluation state jf the respiratory subsystem

The Skibinskaya index is calculated as:

$$
C=x_{0} \frac{x_{1}}{x_{4}}
$$

where $x_{1}$ - time of breath exhalation;

$x_{4}-$ heart rate.

The Skibinskaya index characterizes not only the potential of the system of external respiration, its resistance to hypoxia, but also, to a certain extent, the level of coordination of the respiratory subsystem with the cardiovascular subsystem of the organism.

The index of hypoxia is traditionally calculated by the formula:

$$
B=\frac{x_{1}}{x_{2}}
$$

This calculated indicator characterizes the degree of resistance of the body to oxygen deficiency.

The respiratory system has close links with the cardiovascular system. So, the amount of respiratory volume is directly related to the frequency of breathing and cardiac contractions. Therefore, heart rate and time of breath exhalation.

After transformations (1) the mathematical model has the following form:

$$
Y^{*}=100 \cdot \frac{x_{1} \cdot\left(x_{3}-x_{2}\right)}{x_{4} \cdot x_{3}}
$$

where $x_{3}=A-$ maximum ventilation of the lungs.

The mathematical equation (5) is the basis for constructing a generalized information model for decisionmaking on the state of the respiratory system shown in Figure 4.

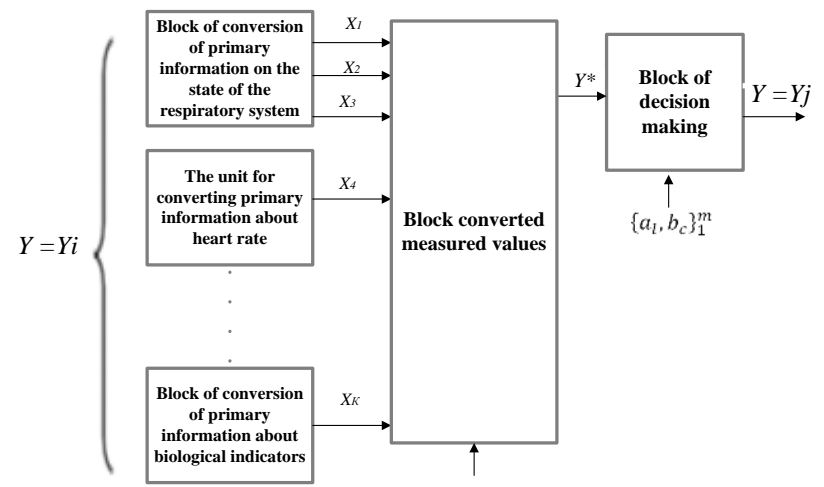

Figure 4. A generalized information model for decision making

The transformation unit for measured values (measurements) converts measured values $x_{1}, \ldots x_{k}$ of controlled values using the SP100 HEACO spirograph and the OMRON MIT3 tonometer.

The proposed model allows you to control more indicators. However, when conducting experimental studies of the status of participants in the Antarctic expedition, only the values $x_{1}, \ldots x_{4}$ were measured.

The conversion unit of the measured values makes the transformation $x_{1}, \ldots x_{4}$ in the $Y^{*}$ estimate of the parameter $Y$ according to the mathematical model:

$$
\mathrm{M}[Y]=F\left(\mathrm{M}\left[X_{1}\right], \ldots \mathrm{M}\left[X_{k}\right]\right) .
$$

The assessment of the respiratory system is carried out at the stage of studying the diagnostic object by the sample size $\mathrm{n}$ for each of the fixed values $Y_{j}, j=\overline{1, m}$ ) of the levels of the parameter $Y$.

The decision-making block selects $\left\{a_{1}, b_{c}\right\}_{1}^{m}$ of one $\mathrm{y}_{\mathrm{j}}\left\{\mathrm{a}_{\mathrm{l}}, \mathrm{b}_{\mathrm{c}}\right\}_{1}^{\mathrm{m}}$ from many $\left\{\mathrm{y}_{\mathrm{l}}\right\}_{1}^{\mathrm{m}}$ solutions of the value $Y$ (Figure 2), after comparing $Y^{*}$ with the norm $\left(\mathrm{a}_{1}, \mathrm{~b}_{1}\right), \mathrm{l}=$ $\overline{=1, \mathrm{~m}}$ in accordance with the rule of decision [13]

$$
\forall Y^{*}\left[Y^{*} \in\left(\mathrm{a}_{\mathrm{j}}, \mathrm{b}_{\mathrm{j}}\right) \rightarrow Y^{*} \in Y_{j}\right]
$$

The equation (1) is also used as a model for calculating the total uncertainty of the measurement of respiratory state.

\section{EVALUATION OF UNCERTAIN}

The main significance for biomedical research is uncertainty, which manifests itself in the mutual influence on each other of the biological object and technical means, the inaccuracy of the implementation of the experimental method, the variability of the properties of the object in the study process, the noise of internal and external origin.

Other sources of uncertainty may be:

- unrepresentative sampling;

- Inadequacy of used models;

- influence of the parameters of the external environment

- insufficient study of input quantities;

- possible correlations of parameters;

- qualifications of experts and operators.

A other task is the analysis of the uncertainty of measuring the indicators describing the state of individual subsystems and the whole organism, as well as the 
uncertainty associated with the accuracy of medical devices that carry out the process of conversion and measurement of biological indicators, as well as taking into account the group and intergroup variability of biological indicators.

The uncertainty calculation was performed on the recommendations of the international document [14]. The basis of the calculation is the monitoring of the status of the pulmonary subsystem of 8 polar explorers, integrated into the reference group for 6 months in the conditions of Antarctica during the performance of official duties. The influence of conditions associated with the expedition's place of residence and influence on the hemodynamics of an organism (increased pressure at low temperatures, a decrease in relative humidity, which leads to an increase in the partial pressure of oxygen in inhaled air, etc.) are taken into account $[15,16]$.

An estimation of the integral index y of the respiratory system is calculated for (5) for the arithmetic mean values $x_{1 \ldots} x_{4}$ :

$$
Y^{*}=100 \frac{x_{1} \cdot\left(x_{3}-x_{2}\right)}{x_{4} \cdot x_{3}}=65,18
$$

Based on the obtained statistical material, estimates of standard uncertainty for type A for each of the measured indicators $x_{1}, \ldots x_{4}$ are calculated using the formula:

$$
u\left(x_{i}\right)=\sqrt{\frac{1}{n(n-1)} \sum_{i=1}^{n}\left(x_{i}-\overline{\bar{x}}_{l}\right)^{2}}
$$

where $\bar{x}_{i}=\frac{1}{\mathrm{n}} \sum_{i=1}^{n} x_{i}$

The arithmetic means of the number of observations for $x_{1} \ldots x_{4}$ are:

$$
\overline{x_{1}}=50,62 ; \overline{x_{2}}=6,79 ; \overline{x_{3}}=113 ; \overline{x_{4}}=73 \text {. }
$$

The values of standard uncertainties of type $A$ for indicators $x_{1} \ldots x_{4}$ are:

$$
\begin{aligned}
& u\left(x_{i_{1}}\right)=1,426 ; u\left(x_{i_{2}}\right)=0,148 ; \\
& u\left(x_{i_{3}}\right)=2,26 ; u\left(x_{i_{4}}\right)=1,8898 .
\end{aligned}
$$

Standard uncertainties of type B associated with measurement accuracy of the measuring instruments SP100 HEACO spirograph and OMRON MIT3 tonometer are:

for heart rate:

$$
u_{B_{4}}\left(x_{i_{4}}\right)=0,03
$$

for values $x_{1}, \ldots x_{3}$ :

$$
u_{B_{1 . .3}}\left(x_{i_{1 . .3}}\right)=0,06
$$

To estimate the combine standard uncertainty, the $c_{i}$ sensitivity coefficients for each input value are calculated and their contributions $u\left(y_{i}\right)$ are calculated in the total standard uncertainty

for $x_{1}$ :

$$
u\left(y_{1}\right)=\frac{x_{3}-x_{2}}{x_{4} \cdot x_{3}} \cdot \sqrt{u_{B_{1 . .3}}\left(x_{i_{1.3}}\right)^{2}+u_{A_{1}}\left(x_{i_{1}}\right)^{2}}=0,018
$$

for $x_{2}$ :

$$
u\left(y_{2}\right)=\frac{x_{1}\left(x_{3}-1\right)}{x_{4} \cdot x_{3}} \cdot \sqrt{u_{B_{1 . .3}}\left(x_{i_{1.3}}\right)^{2}+u_{A_{2}}\left(x_{i_{2}}\right)^{2}}=0,1099
$$

for $x_{3}$ :

$u\left(y_{3}\right)=\frac{x_{1}\left(1-x_{2}\right)}{x_{4} \cdot\left(-x_{3}\right)^{2}} \cdot \sqrt{u_{B_{1 . .3}}\left(x_{i_{1 . .3}}\right)^{2}+u_{A_{3}}\left(x_{i_{3}}\right)^{2}}=0,000817$

for $x_{4}$ :

$$
u\left(y_{4}\right)=\frac{x_{1}\left(x_{3}-x_{2}\right)}{x_{3} \cdot\left(-x_{4}\right)^{2}} \cdot \sqrt{u_{B_{4}}\left(x_{i_{4}}\right)^{2}+u_{A_{4}}\left(x_{i_{4}}\right)^{2}}=0,032
$$

The combine standard uncertainty is:

$$
u_{c}(y)=\sqrt{\sum_{i=1}^{m} u_{i}^{2}(y)}=0,126
$$

Extended uncertainty is at $\mathrm{p}=0.95$ :

$$
U= \pm 0,25 \text {. }
$$

Consequently, without taking into account individual variability and between the group variability of the indicator, only due to the imperfection of their measurement, the result can be presented as:

$$
Y=(65,18 \pm 0,25) \text { at } \mathrm{p}=0,95
$$

To make a decision on the state of the organism, it is necessary to take into account the individual $V_{i}$ and inter individual variability of $V_{G}$. For example, for the considered group, $V_{i}$ lung capacity can fluctuate within $15 \%, V_{G}-$ within $14 \%$; Skibitsky index $V_{i}$ - within $24 \%, V_{G}$ - within $33 \%$; heart rate for 5 minutes $V_{i}$ - within $8 \%, V_{G}$ - within $10 \%$ [17].

The uncertainty of the influence of factors in assessing the indicator and ensuring the required level of reliability of the decision on the state of the organism's system should be met by inequality:

$$
U<0,25\left(V_{i}^{2}+V_{G}^{2}\right)^{1 / 2}
$$

To fulfill this inequality, one should especially carefully prepare the experiment and strictly adhere to the method of its implementation in order to eliminate the influence of systematic effects on the results obtained.

\section{CONCLUSION}

The reliability of making decisions about the functional state of such a complex system as a biological object requires a comprehensive analysis of the obtained medical and biological indicators. In addition to estimating the uncertainty of the indicators obtained during the measurement, it is necessary to take into account such 
specific features as the individual and intergroup variability of the object itself.

The rule of making a decision on the functional state of all subsystems of a biological object, taking into account the uncertainty of its indicators, will provide a basis for determining the integral indicator of the state of the entire body system. The value of the integral index provides the basis for the correct use of all types of resources, both material and labor, as well as the ability to predict the likelihood of professional duties, especially in extreme conditions. Extreme factors, affecting the change in the state of the functional equilibrium of the organism (homeostasis), can lead to disruption of the work of the entire expedition.

Prognosis of the functional state of a biological object is an open question for future decisions.

\section{REFERENCES}

[1] Bemmel J. H. Handbook of medical informatics / J. H. van Bemmel, M. A. Musen. - Houten : Springer-Verlag, -628 p., (2002).

[2] Bronzino J. D. Medical Devices and Human Engineering Four Volume Set / J. D. Bronzino, D. R. Peterson. - Boca Raton : CRC Press. -563 p. , (2014).

[3] V.D. Kuzovyk, A.D.Gordieiev, I.I. Burdenyuk, M.Maciejewski \&S.Kalimoldayeva. Bioengineeringsystem for professional recruiting and predictionof psihological changes in the bodyof extreme activitiesoperators.// Information technology in medical diagnostics. II. 2019, pp.59-66. ISBN 9780367177690.

[4] Y.Moiseyenko. V.Sukhorukov, G.Pyshnov, I.Mankovska, K.Rozova, and other. Antarctica chalenges the new horizons in predictive, preventive, personalized medicine: preliminary results and attractive hypotheses for multi-disciplinary prospective studies in the Ukrainian “Akademik Vernadsky" station // EPMA Journal. - 2016. - T 7, №11. P.1-19.

[5] Family Medicine: Principles and practice / R. B. Taylor, J. L. Buckingham, E. P. Donatelle, T. A. Johnson, Jr., J. E. Scherger. New York : Springer-Verlag. -743 p., (1988).
[6] Pisanelli D. M. Ontologies in Medicine / D. M. Pisanelli. Amsterdam : IOS Press. - 165 p., (2004)

[7] Webster J. G. Medical instrumentation. Application and design / J. G. Webster - New York : John Wiley \& Sons. - 675 p., (2009).

[8] Ledzhevich, U. (ets.) Mathematical Methods and Models in biomedicine. - Springer, (2013).

[9] Hall, John (2011). Guyton and Hall textbook of medical physiology (12th ed.). Philadelphia, Pa.: Saunders/bich er. pp. 4 9781416045748

[10] Vlasov. V.V. Epidemiologiya: tutorial-- M.: GOETAR-Medb 2004.$464 \mathrm{~s}$.

[11] E. Volodarsky, Z.Warsza, L.Kosheva, A. Idźkowski improve accuracy of the chromatography mass spectrometry automation - 2016:conference Challenges in Automation, Robotics and Measurement Techniques. series "Advances in Intelligent Systems and Computing", Springer, March 2-4, 2016,-

[12] Bayevskiy R.M., Berseneva A.P. Introduction to prenosological diagnosis. M.: Slovo, 2008. $220 \mathrm{~s}$.

[13] Jonhson N., Leone F.: Statistical and experimental design. Engineering and the Physical Science. vol. 2, second ed. Wiley and Sons. New York-London-Sidney-Toronto, (1977).

[14] ISO/IEC Guide 98-1:2009 1:2009 (JCGM/WG1/104) Uncertainty of measurement - Part 1: Introduction to the expression of uncertainty in measurement.

[15] Barnett A.G., Sana S., Solomaa V., Kuulasmaa K., Dobson A.J. The effect of temperature on systolic blood pressure // Blood Press. Monit.2007, №. 3. P. 195-203

[16] Madsen C., Nafstad P. Assosiation between environmentalethedsute and blood presser among participant in the Oslo Health snallysigy Eur.J.Epidemiol.2006. Vol.21, № 7. P. 485-491

[17] Yu.G. Solonin, A.L. Markov, YE.R. Boyko. Biology. Earth Sciences . 2012. Issue. 1, pp. 103-108 